\title{
Part 4
}

Massive Star Formation 


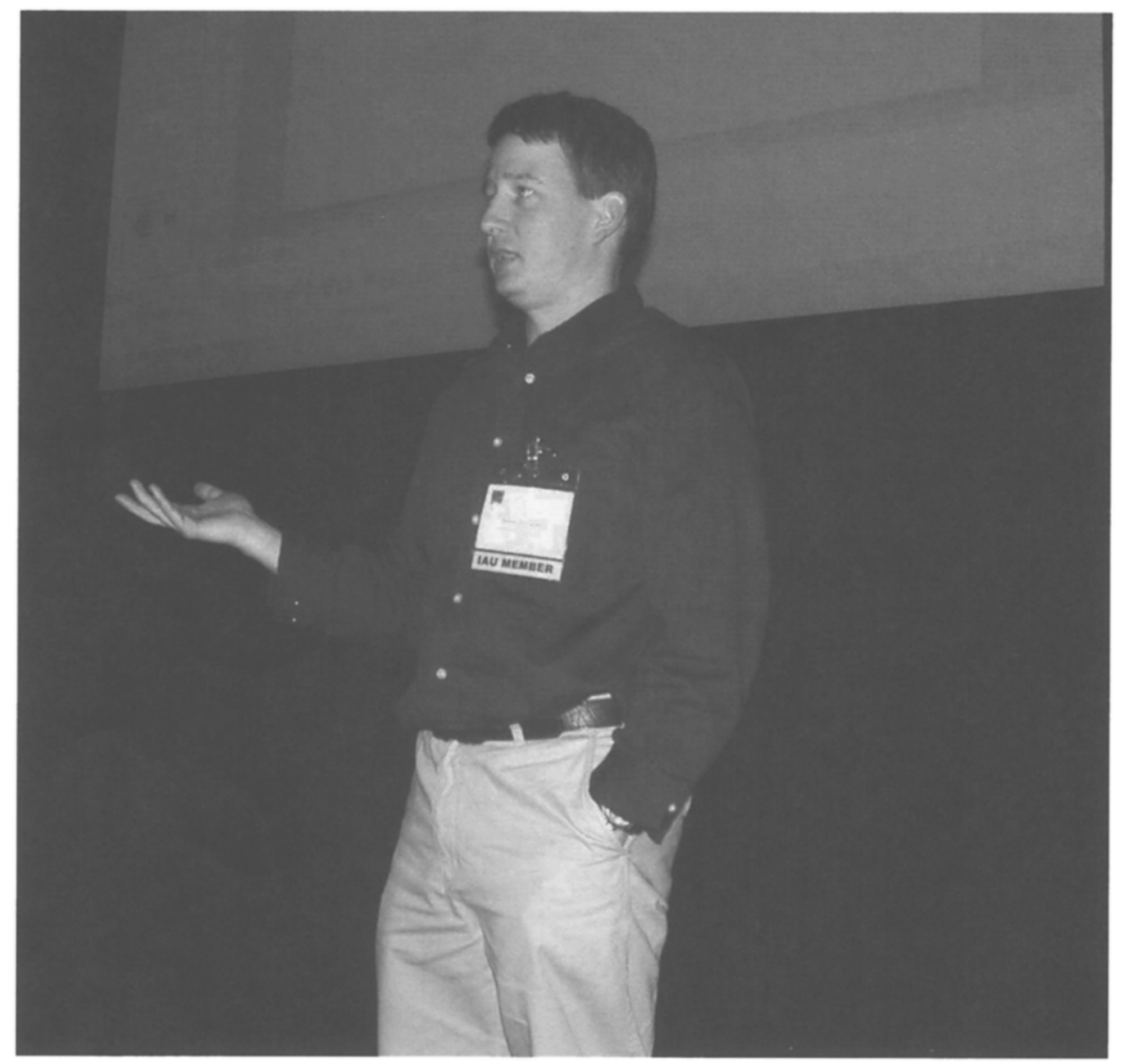

Simon Ellingsen 


\title{
Masers: High resolution probes of massive star formation
}

\author{
S P Ellingsen
}

School of Mathematics and Physics, University of Tasmania, Private Bag 37, Hobart 7001, TAS, Australia

\begin{abstract}
Astrophysical masers are one of the most readily detected signposts of high-mass star formation. Their presence indicates special conditions, probably indicative of a specific evolutionary phase. Masers also represent the ultimate high-resolution probe of star formation with the potential to reveal information on the kinematics and physical conditions within the region at milli-arcsecond resolution. To date this potential has largely remained unfulfilled, however, recent advances suggest that this will soon change.

The key to unlocking the potential of masers lies in identifying where they fit within the star formation jigsaw puzzle. I will review recent high resolution observations of $\mathrm{OH}$, water and methanol maser transitions and what they reveal. I also briefly discuss how multi-transition observations of $\mathrm{OH}$ and methanol masers are being used to constrain maser pumping models and through this estimate the physical conditions in the masing region.
\end{abstract}

\section{Introduction}

Masers are one of the most readily observable signposts of massive star formation and they possess characteristics that mean they have the potential to be very powerful high-resolution probes of these regions. Towards massive star forming regions strong masers are frequently observed from the main-line transitions of $\mathrm{OH}$ (Caswell, Haynes \& Goss 1980), the $22 \mathrm{GHz}$ transition of $\mathrm{H}_{2} \mathrm{O}$ (Comoretto et al. 1990) and the 6.7 GHz (Menten 1991) and 12.2 GHz (Batrla et al. 1987) transitions of methanol. They are more rarely observed from $\mathrm{SiO}$ (see Greenhill et al. these proceedings), $\mathrm{H}_{2} \mathrm{CO}$ (Hoffman et al. 2003) and $\mathrm{NH}_{3}$ (Mauersberger Wilson \& Henkel 1986) and from other transitions of $\mathrm{OH}, \mathrm{H}_{2} \mathrm{O}$ and methanol.

The presence of maser emission indicates that the physical conditions within the region lie within a certain range. However, despite the fact that the first masers were discovered towards star forming regions nearly 40 years ago (Weaver et al. 1965) the precise physical conditions that give rise to the various maser transitions in massive star forming regions are still not understood. There are two fundamental reasons for this, the first is that the pumping of the masers is an intrinsically complex and non-linear process. The second reason is that aside from information obtained directly from observations of the maser itself (such as intensity, brightness temperature, line width and polarization properties) there is typically little or no complementary information available at 
resolutions comparable to the size of the masers. Towards some star formation regions multiple different $\mathrm{OH}$ maser and/or methanol maser transitions are detected. If these transitions arise from the same region of gas then this places significant additional constraints on maser pumping schemes. To date multitransition observations of OH (Cesaroni \& Walmsley 1991, Gray et al. 2001) and methanol (Cragg et al. 2001, Sutton et al. 2001, Ellingsen et al. 2003) masers have only been made towards a small number of sources. Refinements in observational techniques and maser modeling offer the possibility that future multi-transitional observations may yield vital information on the physical conditions in star-formation regions at high-resolution.

The most common $\mathrm{OH}$, water and methanol maser transitions have been detected towards hundreds of massive star forming regions within the Galaxy and many regions show emission from all three species. The exact relationship between the various maser molecules, and the environment and evolutionary phase that they each trace is currently understood at only a very superficial level. Studies of the relationship between $\mathrm{OH}$ and water masers has found that in regions where these two transitions coexist they arise in nearby (separations of less than a few arcseconds), but different locations (Forster \& Caswell 1989). A detailed study of methanol and water masers by Beuther et al. (2002) found a similar relationship for these two species. In contrast towards many highmass star forming regions there is a close association between the presence and location of $\mathrm{OH}$ and methanol masers and initially most methanol masers were discovered towards known $\mathrm{OH}$ masers. High resolution studies have found that in many cases the clusters of $\mathrm{OH}$ and methanol masers are coincident at the subarcsecond level (Caswell 1997), but individual $\mathrm{OH}$ and methanol maser spots arise at differing locations (Menten et al. 1992). However, there are many methanol and water maser sites which are not associated with $\mathrm{OH}$ masers and untargetted searches for methanol masers have detected many not associated with known star formation regions (Ellingsen et al. 1996). In addition, centimetre radio continuum observations towards a large sample of methanol masers have also failed to detect emission stronger than $1 \mathrm{mJy}$ towards the majority (Phillips et al. 1998, Walsh et al. 1998), although all are associated with millimeter continuum sources (Beuther et al. 2002). The generally accepted current picture is that water and methanol maser emission commences prior to the formation of a detectable ultra-compact $\mathrm{HII}$ (UCHII) region, with the $\mathrm{OH}$ masers arising after the commencement of fusion in the core. There is a period during which all three species can coexist, but relatively soon after the formation of a detectable UCHII the methanol masers are destroyed, followed by the water masers with the $\mathrm{OH}$ masers surviving until later in the evolutionary process. A more detailed discussion of the relationship between the various maser species in star formation regions is given in Caswell (2002) and Beuther et al. (2002).

\section{Masers - the ultimate high resolution probes}

As stated above, the presence of a maser within a star forming region indicates the existence of particular physical conditions. In most regions where masers are detected it is likely that there are large volumes of gas in the right physical condition to mase, but we only observe a maser where by chance there is a 
long path which exhibits a large degree of velocity coherence along our line of sight. Under this picture there is nothing special about our particular line of sight and observers along different lines of sight will just detect maser emission from different locations within the larger masing region. It also explains why the masers are intrinsically small. Being both small and bright means that it is possible to image masers using Very Long Baseline Interferometry (VLBI) and as they are spectral lines we are able to obtain both the relative position and light of sight velocity of the different maser spots. It is often possible to determine the positions of the different maser spots within a region to sub milliarcsecond (mas) accuracy relative to a chosen reference maser spot or to some nearby background continuum reference source. To put this in perspective, for a massive star formation region at a distance of $2 \mathrm{kpc}$ an angular resolution of 1 mas corresponds to a linear resolution of $0.3 \mathrm{AU}$. The small size of the masers and the high accuracy to which their relative positions can be determined means that it is possible to measure proper motions of the masers on time-scales as short as weeks, but more typically on time-scales of years (e.g. Genzel et al. 1981a). This makes masers very powerful probes of the kinematics in the regions where they exist. To date, it is as probes of the kinematics that masers have made the most striking contribution to our understanding of massive star formation at high-angular resolution and section 4 discusses some specific examples of this.

Masers are also able to yield information on the magnetic fields within the regions where they form. The $\mathrm{OH}$ molecule is paramagnetic and this means that it undergoes significant Zeeman splitting in the presence of interstellar magnetic fields. The Zeeman splitting causes $\mathrm{OH}$ masers to be highly circularly polarized, sometimes up to $100 \%$. VLBI observations can be used to identify Zeeman pairs (left and right-hand circularly polarized $\mathrm{OH}$ masers with different velocities that are coincident at the milli-arcsecond level) and the total magnetic field strength in the masing region can then be determine from the velocity difference between the two orthogonal polarizations (see for example Caswell (2002)). The size of the velocity separation for the Zeeman pairs depends upon the Lande-g factor for the transition and differs for each $\mathrm{OH}$ maser transition. The excited $\mathrm{OH}$ transitions at 6030 and $6035 \mathrm{MHz}$ have both smaller Zeeman splitting and generally simpler spectra than the main-line transitions which means that it is possible to confidently identify orthogonal polarization pairs from single dish spectra alone, particularly when observations are made of more than one transition (Caswell 2003).

\section{Masers - the Bart Simpson of star formation research}

If as outlined in the previous section masers are such powerful probes, then it is reasonable to ask why they are not more prominent in the field of star formation research? Studies of masers play a prominent role in studies of AGB and postAGB stars and maser observations have been the key to a number of new and exciting discoveries relating to active galaxies. So why is it that for star forming region, masers (like Bart Simpson) are under-achievers? I believe that the most important factor is that in contrast to AGB stars and active galaxies there is relatively little complementary high-resolution information at other wavelengths available for star formation regions. The earliest stages of high-mass star forma- 
tion take place deep within cold, dense clouds of gas and dust which are often optically thick at wavelengths as long as $20 \mu \mathrm{m}$. In addition, high-mass stars form in clusters and so high resolution observations are essential to disentangle the different phenomena associated with stars of a variety of masses at a various evolutionary phases. High resolution observations in the mid and far-infrared and sub-millimeter regimes are only now becoming a reality and these are set to revolutionize our understanding of massive star formation. Far-infrared and sub-millimeter images of high-mass star formation at arcsecond resolution will help to resolve the confusion of the cluster environment and characterise the region in which the masers arise. I believe that a better understanding of the environment in which the masers form will unlock their potential as probes of high-mass star formation, much as it has for AGB stars and active galaxies.

\section{Masers as high resolution probes}

There are a number of sources where studies of the proper motion of masers in high-mass star formation regions have made a significant contribution to our understanding of the kinematics. The rest of this paper will detail some of the best examples of masers as kinematic probes.

\subsection{Water masers in Cepheus $A$}

In the late 1970's and early 1980's the first VLBI proper motion observations of water masers were undertaken towards sources like Orion KL and W51 (Genzel et al. 1981a,b). A relatively common feature of water maser spectra in massive star forming regions is strong emission at the systemic velocity and weaker emission at velocities offset by $\pm 50-200 \mathrm{~km} \mathrm{~s}^{-1}$. The observations of Genzel et al. revealed that the systemic velocity features typically show large proper motions, while the high-velocity features show much smaller proper motion. This is readily explained if the water masers arise in regions of post-shocked gas. If the direction of propagation of the shock is close to the plane of the sky then the line of sight velocity will be approximately the systemic velocity, but the transverse velocity and hence the proper motion will be large. Conversely if the shock is propagating in a direction close to the line of sight then the velocity of the maser emission will be offset from the systemic velocity of the region, but the proper motion will be small. The path length through the post-shocked gas is also much smaller in this case which explains why the high-velocity maser features are weaker than those at the systemic velocity. Proper motion experiments enable the three-dimensional velocity distribution of shocks and outflows in high-mass star formation regions to be traced. However, the maps of maser water maser emission and proper motions of Genzel et al. and others show that in practice the overall distribution of masers is complex and since location of protostars, YSO etc. in the regions is typically unknown, interpretation of the maser proper motions is difficult.

There are a small number of water maser sources where it has been hypothesised that the emission arises in a circumstellar disk. Finding unambiguous evidence for maser emission in a disk (the equivalent of NGC4258 for active galaxies), is something of a holy grail for researchers studying masers in star formation regions. There is good evidence for the existence of disks around 
high-mass stars from infra-red and thermal molecular line observations (see for example papers by Padgett and Wilner in these proceedings). Masers offer the possibility of revealing detailed information on the disk dynamics which could be used to infer disk dimensions, whether it is warped by instabilities, accretion rates etc. To date none of the purported disks in star-forming regions are as convincing as that observed towards the nucleus of NGC4258 (Miyoshi et al. 1995), but proper motion observations of a possible disk associated with Cepheus A HW2 by Torrelles et al. (2001) have discovered an even more intriguing object.

The tale of Cepheus A HW2 is a theme common to high resolution observations of masers from a variety of transitions. Observations with arcsecond resolution with a connected element interferometer (in this case the VLA) showed a flattened structure perpendicular to a radio continuum jet with a velocity gradient and this was hypothesised to be a disk (Torrelles et al. 1996). To test the hypothesis further observations using a VLBI array with milli-arcsecond resolution (in this case the VLBA) were made, however, rather than confirming the original hypothesis the higher resolution observations instead revealed greater complexity. Torrelles et al. found that what appeared to be single maser spots at arcsecond resolution were in many cases very narrow linear and curved structures, often showing proper motion perpendicular to the major axis. One of the maser spots slightly resolved at arcsecond resolution is revealed to be a remarkable arc of water masers. The arc of masers extends over 0.1 arcseconds $(72 \mathrm{AU}$ at a distance of $725 \mathrm{pc}$ ), fits a circle with an accuracy of 1 part in 1000 and is expanding with a velocity of $9 \mathrm{~km} \mathrm{~s}^{-1}$. The radius of the circle is $62 \mathrm{AU}$ and it has a dynamical age of 33 years (Torrelles et al. 2001). Recent observations with the VLA have detected a weak radio continuum source coincident with the centre of the expanding circle of masers, although it has not yet been possible to identify the nature of this source (Curiel et al. 2002). The highly circular appearance of the arc of masers is strongly suggestive of spherical expansion and the masers are thought to be due to either spherical episodic ejection from a protostar or; the initial expansion of an HII region from a B3 or B4 star. Either way further study of this object seems set to yield new information on the formation of high-mass stars and their interaction with their natal environment.

\subsection{Methanol masers in NGC7538}

One advantage of observations of class II methanol masers such as the 6.7 and $12.2 \mathrm{GHz}$ transitions is that unlike $\mathrm{OH}$ and water masers that are associated with a range of astrophysical environments, methanol masers are only found towards high-mass star formation regions (Minier et al. 2003). Early arcsecond resolution images of class II methanol masers found that many of them exhibited a simple linear or curved spatial morphology, often with a monotonic velocity gradient along the line. The most popular hypothesis for this observation was that the methanol masers traced an edge-on circumstellar disk (Norris et al 1993, 1998). However, as with the water masers in Cepheus A, the milli-arcsecond resolution images while consistent with the arcsecond resolution images frequently reveal more complex structures which are not easily explained as edge-on disks (e.g. Minier, Booth \& Conway 2000, De Buizer et al. 2002). These and other observations and arguments suggest that the vast majority of methanol masers do not arise in edge on disks, but are more likely to be associated with shocks 
(Lee et al. 2002). Although clearly lower velocity and less energetic shocks than those that produce water masers.

Despite this there are some sources for which the milli-arcsecond resolution images are most easily explained as an edge-on disk. The best example is that of NGC7538 which has a shows a single very narrow emission feature which in the $12.2 \mathrm{GHz}$ transition is approximately 45 mas (110 AU) long with a monotonic velocity gradient along it (Minier, Booth \& Conway 1998). Assuming Keplarian rotation, a measurement of velocity gradient along still has a dependency between the binding mass and the radius of the emission. If a plausible high-mass protostellar mass is assumed for NGC7538 $(10 \mathrm{M} \odot)$ then the implied radius of the disk is of the order of 940 AU (Minier et al. 2000). This implies that either the methanol maser emission traces only a very small fraction of the total disk or that the disk is not that of a high-mass protostar. In general low implied masses or small disk fractions are a plausibility issue for many potential methanol maser disk sources (Minier et al. 2000).

\section{3. $\mathrm{OH}$ masers in $\mathrm{W3}(\mathrm{OH})$}

Like water masers, $\mathrm{OH}$ masers are associated with a range of astrophysical objects, including evolved stars, SNR/molecular cloud interfaces and high-mass star formation regions. $\mathrm{OH}$ masers were first discovered towards star formation regions and the best studied region is $\mathrm{W} 3(\mathrm{OH})$ which exhibits $\mathrm{OH}$ maser emission from a wide variety of transitions, some of which haven't been observed towards any other sources (Cesaroni \& Walmsley 1991). The OH masers (and the class II methanol masers) lie in a number of clusters projected in front and just beyond the edge of an ultracompact HII region (Reid et al. 1980, Menten et al. 1992). As with many/most OH masers there is little or no apparent internal structure to the clusters. Proper motion observations of the $\mathrm{OH}$ masers by Bloemhof, Reid \& Moran (1992) find the masers to be in slowly expanding $\left(3 \mathrm{~km} \mathrm{~s}^{-1}\right)$ gas. This observation supports the generally accepted picture that the $\mathrm{OH}$ masers arise at the interface between the ionized and molecular gas.

An exciting recent development is the detection by Argon, Reid \& Menten (2003) of weak OH maser emission associated with the Turner-Welsh (TW) object near W3(OH). The TW object is a high-mass protostar offset approximately 6 arcseconds from the $\mathrm{W} 3(\mathrm{OH}) \mathrm{UCH}$ II region which has associated water masers and synchrotron jets. The $\mathrm{OH}$ maser emission arises at the end of the water maser emission and may indicate that $\mathrm{OH}$ masers found towards star formation regions which are not directly associated with radio continuum emission, may trace outflows. They also offer the possibility of probing the jets from the TW object and other similar objects at high-resolution in more than one regime (as the $\mathrm{OH}$ maser emission appears to arise further out in the jet than the water masers).

\section{Conclusions}

For a small, but growing number of sources observations of masers are enabling high-mass star formation regions to be studied at sub-arcsecond resolutions. The examples above demonstrate that VLBI proper motion studies are a powerful method for probing the kinematics within the star forming regions, the challenge 
we currently face is to understand exactly what part of the star formation process the masers are associated with. Multi-transition studies of $\mathrm{OH}$ and methanol masers, are currently in their infancy, but offer the prospect of being able to tightly constrain the physical conditions which produce the masers and which will greatly enhance the information obtained from kinematic studies. Combined with complementary high-resolution studies of high-mass star formation regions in the millimeter through to mid-infrared wavelength ranges there appear to be good prospects that in the near future masers will play a much more prominent role in studies of high-mass star formation than is presently the case.

\section{Acknowledgements}

This research has made use of NASA's Astrophysics Data System.

\section{References}

Argon, A. L., Reid, M. J., Menten, K. M. 2003, ApJ, 593, 925

Batrla, W., Matthews, H. E., Menten, K. M., Walmsley, C. M. 1987, Nature, 326,49

Beuther, H., Walsh, A. J., Schilke, P., Sridharan, T. K., Menten, K. M., Wyrowski, F. 2002, A\&A, 390, 289

Bloemhof, E. E., Reid, M. J., Moran, J. M. 1992, ApJ, 397, 500

Caswell, J. L. 1997, MNRAS, 289, 203

Caswell, J. L. 2002 in IAU Symp. 206, Masers: From protostars to Blackholes, ed. V. Migenes \& M. J. Reid (San Fransico: ASP), 1

Caswell, J. L. 2003, MNRAS, 341, 551

Caswell, J. L., Haynes, R. F., Goss, W. M. 1980, Aust J. Phys., 33, 639

Cesaroni, R., Walmsley, C. M. 1991, A\&A241, 537

Comoretto, G., Palagi, F., Cesaroni, R., Felli, M., Bettarini, A., Catarzi, M., Curioni, G. P., Curioni, P., di Franco, S., Giovanardi, C., Massi, M., Palla, F., Panella, D., Rossi, E., Speroni, N., Tofani, G. 1990, A\&AS, 84, 179

Cragg, D. M., Sobolev, A. M., Ellingsen, S. P., Caswell, J. L., Godfrey, P. D., Dodson, R. G. 2001, MNRAS, 323, 939

Curiel, S., Trinidad, M. A., Cantó, J., Rodríguez, L. F., Torrelles, J. M., Ho, P. T. P., Patel, N. A., Greenhill, L. J., Gómez, J. F., Garay, G., Hernández, L., Contreras, M. E., Anglada, G. 2002, ApJ, 564, L35

De Buizer, J. M., Walsh, A. J., Piña, R. K., Phillips, C. J., Telesco, C. M. 2002, ApJ, 564, 327

Genzel, R., Reid, M. J., Moran, J. M., Downes, D. 1981a, ApJ, 244, 844

Genzel, R., Downes, D., Reid, M. J., Moran, J. M., Johnston, K. J., Spencer, J. H., Matveynko, L. I., Kogan, L. R., Kostenko, V. I., Rönnäng, B. 1981b, ApJ, 247, 1039

Gray, M. D., Cohen, R. J., Richards, A. M. S., Yates, J. A., Field, D. 2001, MNRAS, 324, 643 
Ellingsen, S. P., Cragg, D. M., Minier, V., Muller, E., Godfrey, P. D. 2003, MNRAS, 344, 73

Ellingsen, S. P., von Bibra, M. L., McCulloch, P. M., Norris, R. P., Deshpande, A. A., Phillips, C. J. 1996, MNRAS, 280, 378

Forster, J. R., Caswell, J. L. 1989, A\&A, 213, 339

Hoffman, I. M., Goss, W. M., Palmer, P., Richards, A. M. S. 2003, ApJ, in press

Lee, J. -K., Walsh, A. J., Burton, M. G., Ashley, M. C. B. 2001, MNRAS, 324, 1102

Mauersberger, R., Wilson, T. L., Henkel, C. 1986, A\&A, 160, L13

Menten, K. M. 1991, ApJ, L75

Menten, K. M., Reid, M. J., Pratap, P., Moran, J. M., Wilson, T. L. 1992, ApJ, 201, L39

Minier, V., Booth, R. S., Conway, J. E. 1998, A\&A, 336, L5

Minier, V., Booth, R. S., Conway, J. E. 2000, A\&A, 362, 1093

Minier, V., Ellingsen, S. P., Norris, R. P., Booth, R. S. 2003, A\&A, 590, 162

Miyoshi, M., Moran, J., Herrnstein, J., Greenhill, L., Nakai, N., Diamond, P. Inoue, M. 1995, Nature, 373, 127

Norris, R. P., Whiteoak, J. B., Caswell, J. L., Wieringa, M. H., Gough, R. G. 1993, 412, 222

Norris, R. P., Byleveld, S. E., Diamond, P. J., Ellingsen, S. P., Kesteven, M. J., McCulloch, P. M., Reynolds, J. E., Tzioumis, A. K., Takahashi, Y., Troup, E. R., Wellington, K. J. 1998, ApJ, 508, 275

Phillips, C. J., Norris, R. P., Ellingsen, S. P., Rayner, D. P. 1998, MNRAS, 294, 265

Reid, M. J., Haschick, A. D., Burke, B. F., Moran, J. M., Johnston, K. J., Swenson Jr, G. W. 1980, ApJ, 239, 89

Sutton, E. C., Sobolev, A. M., Ellingsen, S. P., Cragg, D. M., Mehringer, D. M., Ostrovskii, A. B., Godfrey, P. D. 2001, ApJ, 554, 173

Torrelles, J. M., Gómez, J. F., Rodríguez, L. F., Curiel, S., Ho, P. T. P., Garay, G. 1996, ApJ, 457, 107

Torrelles, J. M., Patel, N. A., Gómez, J. F., Ho, P. T. P., Rodríguez, L. F., Anglada, G., Garay, G., Greenhill, L., Curiel, S., Cantó, J., 2001, Nature, 411,277

Walsh, A. J., Burton, M. G., Hyland, A. R., Robinson, G. 1998, MNRAS, 301, 640

Weaver, H., Williams, D. R. W., Dieter, N. H., Lum, W. T. 1965, Nature, 208, 29 